\title{
TREINAMENTOS EM EPIDEMIOLOGIA REALIZADOS EM INSTITUIÇÕES CONVENIADAS COM O CENTRO NACIONAL DE EPIDEMIOLOGIA (CENEPI): CONGRUENCIAS E DISCREPÂNCIAS COM INDICADORES *
}

Prof. José Florêncio Rodrigues Jr. Universidade de Brasilia

\section{Resumo}

A avaliação de intervenções na área de saúde pública brasileira é uma prática que vem se consolidando nesses últimos anos. Difcrentes modelos de avaliação têm sido utilizados para cste fim.

O presente estudo destina-se a verificar o grau de atingimento de parâmetros ou referenciais de treinamento cm Epidemiologia estabelecidos pelo Centro Nacional de Epidcmiologia do Ministério da Saúde (CENEPI) por de\% instituições de ensino superior conveniadas por aquele Centro. Para cssc propósito, foi escolhido o modelo de Stake, o qual focaliza-se em congruências e discrẹâncias verificadas na prática das dcr. instituições conveniadas, bem assim na prática dos serviços de saúde envolvidos, em relação aos parâmetros acima referidos.

Os referenciais de treinamento cm Epidemiologia são oito: (a) planejamento conjunto do treinamento, (b) diagnóstico de recursos humanos em Epidcmiologia, (c) avaliação continuada dos convênios, (d)scleção dos candidatos, (c) liberação dos candidatos/modularização dos cursos, (f) instalação de núcleos de Epidemiologia, (g)asscssoramento de cquipcs após treinamento c (h) disscminação de bibliografia de treinamento.

Questionários respondidos pelo CENEPI, instituições de ensino superior c scrviços de saúde mostraram que predomina a congruência entre os três grupos de órgãos com rclação aos oito parâmctros ou referenciais do CENEPI. Entretanto, ressaltam proporções elcvadas de discrepâncias, bem assim de concordâncias quanto ao não atingimento dos referenciais tanto pelas instituiçõcs de ensino supcrior como por serviços de saúde.

\section{Palavras-chave:}

- Avaliação de intervenções em saúde pública:

- Avaliação de treinamento em Epidemiologia:

- Treinamento cm Epidemiologia;

- Parâmetros para treinamentos em Epidemiologia.

Avaliar ć um processo que sc impõc à gestão pública; não menos importante do que o planejar e o executar, o avaliar informa sobre a efícácia c cficiência do que foi realizado. Entretanto, avaliaçõcs necessitam de estrutura teórica que as justifiqucm, sustentem c integrem, sem o que, elas correm o risco de se tomarem inválidas; não poucas vezes, avaliações são prejudicadas por viéses e ambigüidades que tornam seus resulta-

\footnotetext{
* Projeto finaliado pelo CENEPI/MS
}

dos ininterprctáveis e. por consequiência, inútcis.

Numerosos modelos tcóricos têm sido propostos c utilizados na prática de avaliar; entre cles, os de Stufflcbeam (1971). de Scriven (1973) e de Stake (1956; s. d.). Estes c outros modelos tcóricos têm sido cmpregados para avaliar programas de diferentes caracteristicas. A escolha de um modelo para uma avaliaçìo deve scr condicionada pela adequação das características deste às caracteristicas daquela. No caso da presente avaliação de programas de trcinamento em cpidemiologia patrocinados pelo CENEPI. escolheu-se o modclo dc Robert Stakc, também denominado "modelo de discrepância."

Quais razões justificam essa escolha? Em primeiro lugar, o modelo é simples: ele consistc do intcrcruzamento cntre a dimensão temporal do objeto da avaliação - constituida de antecedentes, transaçõcs c resultados - $\mathrm{e}$ da dimensão de contraste (ou discrcpância) entre intenção e realizaçĩo. Em segundo lugar. dada a sua simplicidade, o modelo de Stake é também flcxível, acomodando c.remplares diferenciados do objeto de avaliação. Explicitando, o modelo $\mathrm{cm}$ qucstão permite identificar discrepâncias ou convergências verificadas $\mathrm{cm}$ três momentos de qualquer trcinamento: nos antecedentes, ou seja, na fasc preparatória, nas transações. ou scja, no transcurso do treinamento e. finalmentc. nos resultados do trcinamento. Por outro lado, tendo uma estrutura simples, o modelo pode aplicar-se a diferentes contextos de treinamento; diferentes no que se refere a contcúdos, clientela e contcito de saúde nos quais sc realizam os treinamentos. Essas dimensõcs do modelo de Stakc estão representadas na matriz que seguc:

Intenções

Observações

Antecedentes

Transações

Resultados

Definido o modclo a ser adotado na avaliaçào, seguc-se a pergunta, $\mathrm{O}$ que precisa ser avaliado? A resposta à pergunta ć encontrada no documento do CENEPI, Termo de referência para capacitação em epidemiologia (1992), no qual aquele Centro indica oito referenciais que devem balizar os treinamentos em Epidemiologia resultantes de convênios. Os termos de referência são os seguintes: (a) plançamento conjunto do treinamento, (b) diagnóstico dos recursos humanos 
cm Epidemiologia, (c) avaliação continuada dos convênios, (d) seleção e aproveitamento dos candidatos, (e) libcração dos candidatos/modularização dos cursos, (f) instalação de núcleos de Epidemiologia, (g) assessoramento de equipes após treinamento c (h) disseminação debibliografia do treinamento.

Da justaposição dos componentes do modelo de Stake sobre os referenciais, nomeados anteriormente, resultaram três questionários, constituidos de 54 itens. Os questionários destinam-se às três entidades responsáveis pclos treinamentos: as unidades formadoras de recursos humanos (UFRH), os serviços de saúde (SS) e o próprio CENEPI.

\section{Procedimentos}

A seguir, relata-se sucintamente os procedimentos de operacionalização da avaliação, compreendendo a coleta dos dados, metodologia de tratamento dos mesmos e limitações da avaliação.

\section{Coleta dos Dados}

A operacionalização consistiu no envio, pclo CENEPI, dos questionários às unidades fonmadoras de recursos humanos (UFRH's) conveniadas c aos seniços de saúdc (SS); além dessas cntidades, o próprio CENEPI, representado pela sua Unidade de Apoio ao Desenvolvimento da Epidemiologia (UADE), respondcu o questionário próprio.

Por telefone epor via escrita, o CENEPl manteve contato com as 10 instituições conveniadas, assim como com os serviços de saúde a fim de obter seus questionários respondidos. Foram remetidos os questionários das seguintes instituições:

\section{UFRH SS UADE}

Núcleo de Estudos em

Saúde Coletiva (Recife)

Univ. de São Paulo (São Paulo)

Univ. de São Paulo (Ribeirão Preto)

Univ. Est. de Campinas

Univ. Est. do Rio de Janeiro

Univ. Est. Paulista (Botucatu)

Univ. Fed. da Bahia

Univ. Fed. de Goías

Univ. Fed. do Ceará

Univ. Fed. do Rio de Janciro

Instituições conveniadas às quais foram enviados questionários de avaliação. $O$ sinal $(+)$ indica questionário retornado e o sinal $(-)$ indica questionário não retornado.

Como se pode observar, scte dos dez convênios objetos da presente avaliação completaram os três questionários; aos restantes três faltaram questionários, sẹa da UFRH seja do SS. É necessário salientar que a ausência de dados nesses convênios restringe a abrangência c eficácia da avaliação.

\section{Metodologia}

Recebidos os questionários, o avaliador passou a tabulá-los numa matriz que permitisse observar os dados das três instituições simultaneamente. Essa particularidade é importante à luz do modelo de Stake. vez que nele pretende-se constatar congruências c discrepâncias originárias daquilo que sc intencionou acontecesse e do que cfelivamente foi observado na cxecução dos convênios de treinamento $\mathrm{cm}$ Epidemiologia. A matriz de resumo dos dados seguiu of formato do quadro sumário dos itcns, apresentado na fonnulação do modelo de Stakc, no inicio destc trabalho.

Para que os dados pudessem ser entcndidos pelas instituções participantes da avaliação foi necessário resumi-los, dando-lhes interpretação inteligivel. No presente caso, adotou-se uma convenção da qual se pudesse inferir com o maximo de consistência e integridade as respostas dos qucstionários. Nessa convenção ressaliam dois aspectos: (a) os referenciais explicitados pelo CENEPI para os convênios, cm numero de oito, c (b) a concordância ou discrepância entre os respondentes em cada item. Com base nesses parâmetros, convencionouse assinalar com a Ictra A itens cujas respostas tivessem sido compativcis com o a expectativa do CENEPI $\mathrm{c}$ cujos respondentes tivessem respondido congnientementc. Similarmente, assinalou-sc com a letra B os itens cujas respostas fosscm parcialmente compativeis com a expectativa do CENEPI; isto é, quando um dos respondentes tivesse respondido dando a expectativa como atendida e o outro tivesse dado resposta discrepante deste. Finalmentc. atribui-sc a letra $\mathrm{C}$ a itcns cujos respondentes tenham dado resposta congruente, porćm, deixando de atender ao referencial do CENEPI. Essa convenção foi aprovada pela UADE.

Tabulados os dados com base na convenção descrita antcriormentc, passou-se ao sumário dos mesmos por convênio. Isto é, num quadro resumo representaram-se os oito referenciais de avaliação do CENEPI e o número de ocorrências das catcgorias de avaliação $A$, $\mathrm{B}$, e $\mathrm{C}$, cxplicitadas no parágrafo anterior. Através desse mcio procurou-se fomecer a cada instituição integrante de um convênio um perfil do mesmo à luz dos oito referenciais de avaliaçìo do CENEPI.

\section{Limitações}

Toda avaliação ć suscelivel à influência de fatores de diferentes naturezas. A presente avaliação não foi exceção; ao contrário, cla foi afetada por defcitos, os quais cumpre apontar. Tais defeitos, ć possivel, terão influído no resultado apresentado na avaliação; eles são de três ordens: defeitos do instrumento, de operacionalização e de cronologia.

Os questionários destinados às Unidades Formadoras de Recursos Humanos (UFRH), Serviços de Saúde (SS) c ao próprio CENEPI após terem sido elaborados pelo avaliador, foram submetidos à Unidade de Apoio ao Desenvolvimento da Epidemiologia (UADE) do CENEPI, a qual, por sua vez, os submeteu às UFRH em rcunião. Por conseguintc, os questionários efetivamente enviados àquelas cntidades foram ante- 
riormente validados pelas instituições conveniadas objeto da avaliação. Essc procedimcnto, conquanto útil, não impedc que defcitos de pequeno porte ncles se imiscuam. Por exemplo, alguns itens dos questionários não foram seqüenciados cficientementc, obrigando, dessa forma, o respondentc a seguir uma seqücncia lincar, quando sua resposta poderia remctê-lo a itcns mais adiantados. Tal scqücnciamento ocasiona confusão ao respondente $\mathrm{c}$ prejudica a lógica interna do instrumento. Teria sido desejável, também, quc o próprio avaliador aplicasse o questionário pessoalmente a uma das cntidadcs, o que possivelmente lhe pcrmitiria dar-sc conta de inadequaçooes tais como a refcrida, além de outras; cntretanto. isso não foi possivel.

Ocorrcram tambćm limitaçõcs de ordem operacional. Por cxcmplo, questionários cnviados equivocadamente a instituiçõcs e omissão de página num dos instrumentos; cmbora corrigidos csscs crros, elcs certamentc causam confusão e dispõem o respondente desfavoravelmentc. Finalmente, a cronologia da avaliação foi responsável por limitaçõcs. O periodo durante o qual se processou a avaliação coincidiu, no caso de scis UFRH, com treinamentos ainda não concluídos; essa circunstância repcrcutiu nas respostas, cm particular nos refcrenciais 4 (seleção dos candidatos) c 6 (instalação de núcleos de Epidemiologia). Além disso, é preciso assinalar a ausĉncia de dados relativos a três dos convênios. Esses dados omitidos da avaliação significam, como já foi dito, um fator restritivo à confiabilidade da avaliação como um todo.

As limitações cnumeradas não assumiram, cntretanto, porte que invalide os resultados finais da avaliação. Rcssaltadas, clas alertam os que integram a audiência da avaliação primciro, para a neccssidade dc c.xercer-sc cautela na interpretaçào dos rcsultados; em scgundo lugar, sen cm para que cmprecndimentos futuros busquem critá-las.

\section{Resultados}

Como foi explicitado anteriormente, os dados obtidos através dos questionários consistiam $\mathrm{cm}$ frequîencias de ocorrências das categorias $\mathrm{A}$. B e C cm cada convênio. Por outro lado, o conjunto dos dados originários dos de\% convĉnios suscita uma questão de importância central para o CENEPI, ou sẹa, a distribuição das categorias A, B c C no conjunto dos convênios apresenta difcrenças que seriam atribuiveis a causas ou fatores mais prcpondcrantes do que o acaso? ou, de outro modo, a distribuição daquelas catcgorias dcu-sc de forma alcatória?

Para responder essa questão recorrcu-se a dois procedimentos. Primciro, todas as frequiências das catcgorias $\mathrm{A}, \mathrm{B}$ c C foram convertidas cm proporçōes. Esse procedimento foi motivado pela desigualdade de respostas $\mathrm{cm}$ cada convênio. Segundo, utilizou-se a prova estatistica de Kruskal-Wallis (Sicgel, 1956) para dados não-paramétricos para rcsponder-se adcquadamentc a questão proposta. As proporções de freqücencias das categorias A, B c C nos dez convênios estão apresentadas no quadro a seguir.
Proporções de Freqüências das Categorias A, B e C nos Déz Convênios

\begin{tabular}{|l|c|c|c|}
\hline \multirow{2}{*}{ Convennios } & \multicolumn{3}{|c|}{ Categorias } \\
\cline { 2 - 4 } & A & B & C \\
\hline NESC & 56 & 29 & 15 \\
\hline USP & 29 & 62 & $(19$ \\
\hline USP/RP & $0(1)$ & 60 & 40 \\
\hline UNICAMP & 53 & 27 & \\
\hline UERJ & 55 & 24 & 21 \\
\hline UNESP & 45 & 45 & 10 \\
\hline UFBa & 118 & 55 & 37 \\
\hline UFGO & 45 & 01 & 55 \\
\hline UFCe & 81 & 14 & 115 \\
\hline UFRJ & 55 & 25 & 20 \\
\hline
\end{tabular}

As proporções representadas no quadro acima. tranformadas $\mathrm{cm}$ dados ordinais produziram os scguintes valores: catcgoria A. 189: catcgoria B, $164.5 \mathrm{C}$ categoria $C, 111.5$. Esses números, submetidos ao testc de Kruskal - Wallis, resultou no valor $\$ .77$ para $\mathrm{o}$ indice $\mathrm{H}$.

Consultando-se a tabcla pertincntc. verificon-sc que csse valor de $\mathrm{H}$ corresponde a uma probabilidadc de $p=1$. , ou seja, valores como os que se obtiveram para as catcgorias $A, B$ c $C$ poderiam scr obtidos por acaso $\mathrm{cm} \mathrm{l(0)} \mathrm{de} I()()$ amostras análogas. Tendo-se cm conta quc a pesquisa em Ciências Sociais tem estabclccido o valor $p=.(15$ como limiar definidor de provivel causalidade. cabc rçeitar cssa hipótesc com referĉncia aos dados obtidos na presentc avialiação. Rciterando. a distribuição das catcgorias A.B c C rcsultantes dos dados reunidos dos 10 convênios não se deve a um fator ou causa que tenha atuado sistcmaticamentc no conjunto das instituiçōes conveniadas. Ao contrário. tal distribuição provavelmentc obter-se-ia ordinariamentc $\mathrm{cm}$ situações análogas às da presentc avaliação.

\section{Comentairios}

Nos convénios para treinamento em Epidcmiologia celcbrados entrc o CENEPI $\mathrm{c}$ as $\mathrm{dc}$. Unidades Formadoras de Recursos Humanos predomina a catcgoria A do sistcma de catcgorias adotado na prescnte avaliação. Isso implica di $\%$ cr que, de uma fonna global as instituições cnvolvidas na avaliação - Unidades Formadoras de Recursos Humanos, Scrviços de Saúde e CENEPI-perccbem que os oito referenciais estabclecidos pclo mesmo C.ENEPI para os referidos treinamcntos foram at ingidos. Reportando-sc ao modclo de Stake. chega-sc à conclusâo de que predominam na avaliação congruências cntre o intencionado e o realizado. Esta é a intcrpretação autorizada pclos dados obtidos através dos questionários.

Sc. por um lado, essa ć a interprctaçào derivada da análisc estatística dos dados, por outro lado, a avaliação aponta para uma clevada ocorrência de percepções desencontradas (discrepauntes) cntrc as instituiçōes envolvidas na avaliação quanto aos oito referenciais do 
CENEPI. Isso implica dizer que são numcrosas as ocorrências em que uma instituição - por cxcmplo, a UFRH - afirma que um dado referencial foi atingido $\mathrm{c}$ outra instituição - por exemplo, o SS ou o CENEPI afirmam quc isso não aconteceu ou que ignoram se aconteceu ou não. Este dado alerta para a necessidade de uma comunicação clara, univoca c ininterrupta entre as instituiçōes cnvolvidas nos convênios de treinamento.

É tambćm elcrada a ocorrência de percepçòes congruentes de que um dado referencial de avaliação não foi atingido. Neste caso, embora a congruĉncia das percepçòes tenha se verificado, existe uma clara indicação de que a realidade fícou aquém da expectativa. Este é o caso, por exemplo, de dois indicadores da avaliação dados como não atingidos na quase totalidade dos dez. convênios; foram eles a participação do CENEPI no levantamento dos recursos humanos $\mathrm{em}$ cpidemiologia na localidade do treinamento e a disscminação da bibliografia produzida por uma UFRH cntre as demais. Em ambos os indicadores as informaçōes dão conta de que eles deixaram de ser atingidos na ampla maioria dos convênios.

Em conclusão, há evidências de que o modelo de Stakc, adotado na presente avaliação mostrou-se útil e válido $\mathrm{cm}$ responder questōes relativas à eficácia e eficiência dos treinamentos em epidemiologia rcsultantes de convênios celebrados entrc o CENEPI $c$ as UFRH. Pode-se afirmar, com base nos dados reunidos que predominam os referenciais do CENEPI atingidos nos treinamentos. Entretanto, ao mesmo tcmpo, os dados revelam um número igualmente clevado de percepçòes discrepantes quanto ao atingimento daqueles referenciais, assim como um número clciado de testemunhos coincidentes de que tais referenciais não foram atingidos. Essas são, cerlamentc, áreas requerendo melhoras cm treinamentos futuros.

\footnotetext{
Abstract

Public health authorities have made an increasinglv regular practice to submit interventions in their services to evaluation. Nimmerons evaluation models have been employed to this end. The present study seeks to verify the extent to which training parameters or
}

referents in Epidemiologvestablished by the Epidemionlogy National C'enter (CWNEPI) of the Brazilian Ministry of thealth were attained by ten miversity level institutions contracted by the referred center and also the local public health services. Consonant with this purpose. State's evaluation model was chosen. This model focuses on congruencies and discrepancies occurring in the training proctices of the ten institutions regarding CENEI'S's training paraneters.

The parameters set up by (EWEI' for Iraining in Epidemiology are eight, namelv: (a) joint planning of training, (b) diagnostic of available personnel in epiclemiologv, (c) continmed evaluation of the contracts, (d) screening of candidates, (e) candidate discharge fiom serviceflraining flexibilization, (f) extablishment of state epiclemiologv centers, (g) support to local epidemiology groups after training, and (h) dissemination of training bibliographly:

Ouestionnaires responded by the three groups of institutions involved in the evaluation - CEVEPI, miversity level institutions and local public health services - showed a predominance of congruencies among the three groups of institutions regarding the eight paranenters of training in epiclemiologv. However, discrepancies as well as agreement concerning failure to attain CENEPl's parameters on the part of university level institutions and local public health services were noticeable.

\section{Kev terms.}

- Evaluation in(of) public health interventions;

- Evaluation of training in epidemiology;

- Training in epidemiology:

- Parameters for training in epideniology'.

\section{Referências Bibliográficas}

SIEGEl, S. Estatistica nõo-paramétrica. São Pàulo: Editora McGraw - Hill do Brasil. 1956.

STAKE, R. E. Language, rationalitv, and assessment. In Curriculum evaluation. (s.d.) Termo de referência para capacitação $\mathrm{cm}$ epidemiologia. Ccntro Nacional de Epidemiologia. Ministério da Saídc. 1992.

Endereço do Autor

Faculdade de Educação - UnB

Caiva Postal 3i)8()

Brasília - DF - 7()9 1()-9()() 